Am. J. Trop. Med. Hyg., 65(5), 2001, pp. 657-663

Copyright (C) 2001 by The American Society of Tropical Medicine and Hygiene

\title{
REFERRAL PATTERN OF LEPTOSPIROSIS CASES DURING A LARGE URBAN EPIDEMIC OF DENGUE
}

\author{
BRENDAN FLANNERY, MÔNICA M. PEREIRA, LUCIANA DE FREITAS VELLOSO, \\ CLARISSA DE CASTRO CARVALHO, LINA GÓES DE CODES, GIOVANNA DE SABOIA ORRICO, \\ CIBELE M. RIBEIRO DOURADO, LEE W. RILEY, MITERMAYER G. REIS, AND ALBERT I. KO \\ Department of Public Health Biology and Epidemiology, School of Public Health, University of California, Berkeley, California; \\ Couto Maia Hospital, Secretary of Health for the State of Bahia, Salvador, Bahia, Brazil; Goncalo Moniz Research Center, \\ Oswaldo Cruz Foundation/Brazilian Ministry of Health, Salvador, Bahia, Brazil; Division of International Medicine and \\ Infectious Disease, Weill Medical College of Cornell University, New York, New York
}

\begin{abstract}
During heavy seasonal rainfall in 1996, concurrent epidemics of dengue and leptospirosis occurred in an urban center in northeastern Brazil. We interviewed 110 cases of leptospirosis hospitalized a median of seven days after the onset of illness to evaluate the impact of the dengue epidemic on the triage of suspected leptospirosis from ambulatory clinics to the infectious disease reference hospital. Within the first three days of illness, 46 (42\%) cases sought their first medical evaluation, and 28 (61\% of 46) received a diagnosis of dengue. Dengue diagnoses were associated with a median of five days delay in referral to the infectious disease hospital. Patients who reported initial diagnoses of dengue were more likely than other patients to have required admission to the intensive care unit (odds ratio $[\mathrm{OR}]=2.7,95 \%$ confidence interval $[\mathrm{CI}]=0.8-9.5)$ and to have died during hospitalization $(\mathrm{OR}=5.1,95 \%$ $\mathrm{CI}=0.8-55.0)$. These findings indicate that diagnostic confusion between the early symptoms of leptospirosis and dengue may have contributed to the high mortality observed during the leptospirosis epidemic.
\end{abstract}

\section{INTRODUCTION}

Leptospirosis is a bacterial zoonosis transmitted to humans through contact with urine from infected animals. ${ }^{1}$ Infection with pathogenic Leptospira occurs worldwide and is increasingly recognized as a common cause of acute febrile illness in tropical environments. ${ }^{2,3}$ Clinical manifestations of leptospirosis range from a self-limited febrile illness with headache and muscle pain, to severe, life-threatening disease forms such as Weil's disease, characterized by jaundice, renal failure and bleeding, and severe pulmonary hemorrhage syndrome. ${ }^{4-6}$ Leptospirosis has been traditionally considered to be a rural-based disease. ${ }^{4}$ However, urban epidemics have emerged to become a major public health problem in developing countries. ${ }^{7}$ In Brazil, epidemics of leptospirosis have been reported since the 1960s in major cities such as São Paulo, ${ }^{8}$ Rio de Janeiro, ${ }^{9}$ Recife, ${ }^{10}$ and Salvador ${ }^{11}$ following periods of heavy rainfall. Transmission presumably occurs through contact with floodwater contaminated with urine of infected rats. These urban epidemics occur annually and are associated with severe forms of leptospirosis, resulting in significant mortality. ${ }^{6,12}$

During the rainy season in 1996 in Salvador, a city of more than 2.5 million inhabitants in northeastern Brazil, an outbreak of an illness with jaundice and acute renal failure resulted in more than 300 admissions to the infectious disease reference hospital. Leptospirosis was established as the cause of this outbreak. ${ }^{12}$ Despite aggressive supportive therapy including dialysis and intensive care unit monitoring, mortality from severe leptospirosis was $15 \%$. Antibiotic therapy in leptospirosis is widely believed to provide the greatest benefit when initiated in the early phase of the disease $\mathrm{e}^{1,4,13}$ whereas its efficacy when administered during severe, late-stage disease remains controversial. ${ }^{14,15}$ Therefore, early diagnosis and timely administration of antibiotic therapy are required to reduce leptospirosis mortality during urban epidemics.

As in developing countries throughout Latin America, Brazil has undergone a major demographic transformation as a result of more than $350 \%$ growth in the urban population in the last 40 years. ${ }^{12}$ As a consequence of the expansion of urban slums without basic sanitation, seasonal heavy rainfall and flooding have produced conditions favoring rodentborne epidemic transmission of leptospirosis. The same conditions of rapid urbanization and seasonal rainfall have led to the reintroduction of epidemic dengue fever throughout Latin America. ${ }^{16}$ Coinciding with the 1996 leptospirosis epidemic in Salvador, a dengue epidemic occurred during which more than 20,000 cases of dengue were notified in the city, making it the largest recorded epidemic of dengue in the city of Salvador since the Aedes aegypti eradication campaigns in the 1920s. ${ }^{17}$ The dengue cases were exclusively classic dengue fever and dengue hemorrhagic fever was not reported. ${ }^{17}$ Early-phase leptospirosis and classic dengue fever have overlapping clinical presentations that include non-specific findings such as fever, headache, and muscle pains. Often, it is difficult to differentiate these two diseases prior to the development of late-stage complications of severe leptospirosis. We hypothesized that during the $1996 \mathrm{ep}-$ idemic season, the large number of dengue cases and widespread publicity about the symptoms of dengue led to misdiagnosis of patients with leptospirosis in outpatient clinics and a delay in their referral for treatment to the state infectious disease hospital. Such a delay may have contributed to the severity of the 1996 outbreak of leptospirosis in Salvador. The findings of this study demonstrate the need for close supervision of febrile patients during epidemics of dengue in areas where leptospirosis outbreaks occur.

POPULATION, MATERIALS, AND METHODS

Study population. Active surveillance for cases of leptospirosis was carried out during 1996 at Couto Maia Hospital, the 120-bed reference hospital for infectious diseases for the state of Bahia in Salvador, Brazil. The surveillance case definition was based on specific physical findings (conjunctival suffusion ${ }^{1}$ ) and characteristic late-phase manifes- 
tations of leptospirosis (jaundice and abnormally elevated serum bilirubin levels $[>1.0 \mathrm{mg} / \mathrm{dl}]$ with serum aminotransferase activities less than 20 times the normal upper limit; and evidence for acute renal insufficiency such as oliguria [daily urine output $<500 \mathrm{ml}$ ], abnormally elevated serum creatinine $[>1.5 \mathrm{mg} / \mathrm{dl}]$ or blood urea nitrogen $[>75 \mathrm{mg} /$ dl]). ${ }^{12}$ Patients were excluded if they lived outside of the city of Salvador, had radiologic or laboratory evidence of another disease, were discharged from the hospital with a diagnosis other than leptospirosis, refused to participate, or could not be interviewed due to altered mental status changes related to their illness. After obtaining informed consent, blood and urine samples were collected from study patients for laboratory confirmation of leptospirosis.

Ethical considerations. The research was approved by the institutional review board of Couto Maia Hospital, the Committee for Ethical Research at the Gonçalo Moniz Research Center, FIOCRUZ/Brazilian Ministry of Health in Salvador, Brazil, and by the Committee on Human Rights in Research from the New York Hospital-Cornell Medical Center Institutional Review Board in New York. Informed consent was obtained from all adult participants and from parents or legal guardians of minors.

Data collection. Trained interviewers administered standard questionnaires to obtain information on demographic data, symptoms and their duration, and diagnosis and treatment received during health center visits prior to their hospitalization at the study site. Interviews were completed with parents of four patients (minors) and the family members of three patients with mental status alterations caused by the illness. Interviews were performed on the day of admission for $18(16 \%)$ patients, the following day for $45(41 \%)$ patients, the third day of hospitalization for $32(29 \%)$ patients, the fourth day of hospitalization for $14(13 \%)$ patients, and the fifth day of hospitalization for one (1\%) patient. To assist patients in accurately describing symptoms experienced before admission to the hospital, interviewers asked separately about the time of onset of each of eleven symptoms: fever, headache, generalized muscle pain, calf pain, abdominal pain, decreased urine volume, coughing blood, generalized rash, yellowing of eyes or skin, bilateral reddening of eyes suggestive of conjunctival suffusion, and light-stimulated visual discomfort or pain. Physical examinations and medical chart reviews were performed by the study team to obtain information on clinical and laboratory findings at the time of hospitalization, treatment, and clinical outcome.

Laboratory confirmation of leptospirosis and definitions. Cases were confirmed bacteriologically if Leptospira were cultured from patients' blood or urine and observed by dark field microscopy. ${ }^{18}$ Cases were confirmed serologically with the microscopic agglutination test (MAT) that incorporated the use of 25 reference serovars representing 18 pathogenic serogroups and two nonpathogenic serovars of Leptospira. ${ }^{12,18}$ Serologic confirmation was defined as a fourfold increase in MAT titer between paired sera or a reciprocal MAT titer greater than 1:800 in any serum sample. ${ }^{18}$ Cases were defined as probable if the MAT demonstrated a reciprocal titer greater than 1:100 in one or more serum samples to any serovar.

Types of ambulatory services were divided into five categories: 1) emergency rooms in hospitals administered by the Health Secretary of the State of Bahia, Brazil, 2) public sector 24-hr urgent care centers, 3) emergency rooms in private or non-governmental organization hospitals, 4) public sector clinics or health posts, and 5) clinics not belonging to the public sector system. All patients were able to identify the type of ambulatory service when they did not remember the name of the facility where they sought treatment.

Statistical analysis. For spot mapping, patient resident address was located and coded within one of 1,761 census tracts for the city of Salvador (average area $=0.17 \mathrm{~km}^{2}$; average population $=1,200$ residents). ArcView GIS software (Version 3; Environmental Systems Research Institute, Redlands, CA) was used to map the residence of cases according to census tracts defined by the Brazilian Institute of Geography and Statistics. A time-line of ambulatory visits was created retrospectively from the date of admission to the hospital. The analysis of self-reported symptoms was used to reconstruct a sequence of events. Data were entered and analyzed with EpiInfo (Version 6.04c; Centers for Diseases Control and Prevention, Atlanta, GA). The chi-square test with Yates' correction or Fisher's exact test was used to compare proportions. The non-parametric Wilcoxon rank sum test was used to test for differences in continuous variables. Odds ratios (ORs) and their $95 \%$ confidence intervals (CIs) were calculated to contrast proportions. Statistical significance was defined as a two-sided $P$ value $<0.05$.

\section{RESULTS}

Between April 15 and August 2, 1996, 124 hospitalized patients met the surveillance case definition of leptospirosis. Among cases, overall mortality was $11 \%$ (14 deaths). A total of 14 patients (11\% of 124$)$ could not be interviewed because of disorientation $(n=7)$ or death $(n=7)$ prior to identification. Interviews were conducted prospectively with 110 patients, all of whom had a hospital discharge diagnosis of leptospirosis. Of these, $81(74 \%)$ were laboratory-confirmed $(\mathrm{n}=68 ; 62 \%)$ or probable cases $(\mathrm{n}=13 ; 12 \%$; Table 1). Serologic confirmation could not be completed in 23 (79\%) of the 29 unconfirmed cases because paired serum samples were not obtained. Among the 71 patients with suspected leptospirosis for whom paired samples were collected, the positive predictive value for the surveillance definition was $92 \%$. Confirmed/probable and unconfirmed cases did not differ significantly with respect to demographic characteristics, geographic residence in the city of Salvador, outpatient triage, and clinical presentation on hospitalization (Figure 1A and Table 1), with the exception of a lower proportion of jaundice for unconfirmed cases.

By the time of their admission to the study hospital, patients had developed severe manifestations of late-stage leptospirosis (Table 1). The 110 interviewed patients reported a median of seven days of symptoms prior to admission. Frequent complications included hepatic dysfunction, acute renal insufficiency, hemorrhage, and respiratory distress. Patients required hospitalization for a median of 7.5 days (range $=0-41$ days) and $15 \%$ were admitted to the intensive care unit. Peritoneal dialysis was administered to $17 \%$. The case fatality rate among the 110 interviewed patients was $6 \%$ (7 deaths); the significantly higher number of deaths 
TABLE 1

Characteristics of 110 cases of leptospirosis during their initial outpatient evaluation and subsequent hospitalization, according to laboratory confirmation

\begin{tabular}{|c|c|c|c|c|}
\hline \multirow[b]{2}{*}{ Characteristics } & \multicolumn{2}{|c|}{$\begin{array}{l}\text { Confirmed or probable cases } \\
\qquad(\mathrm{n}=81)\end{array}$} & \multicolumn{2}{|c|}{$\begin{array}{l}\text { Unconfirmed cases } \\
\quad(\mathrm{n}=29)\end{array}$} \\
\hline & $\begin{array}{l}\text { No. of } \\
\text { responses }\end{array}$ & $\begin{array}{c}\text { No. }(\%) \text { or } \\
\text { median (range) }\end{array}$ & $\begin{array}{l}\text { No. of } \\
\text { responses }\end{array}$ & $\begin{array}{c}\text { No. (\%) or } \\
\text { median (range) }\end{array}$ \\
\hline \multicolumn{5}{|l|}{ Demographics } \\
\hline Male gender & 81 & $71(88)$ & 29 & $25(86)$ \\
\hline Median age in years & 81 & $32(5-77)$ & 29 & $33(2-71)$ \\
\hline \multicolumn{5}{|l|}{ Initial outpatient visit } \\
\hline Days of symptoms prior to initial outpatient visit & 79 & $4(0-21)$ & 26 & $3.5(0-9)$ \\
\hline Presumptive diagnosis & 81 & & 29 & \\
\hline Dengue & & $29(36)$ & & $10(34)$ \\
\hline Leptospirosis or hepatitis & & $22(27)$ & & $13(45)$ \\
\hline Other & & $30(37)$ & & $6(21)$ \\
\hline \multicolumn{5}{|l|}{ Hospital admission } \\
\hline Days of symptoms prior to hospitalization & 81 & $7(1-21)$ & 29 & $6(3-15)$ \\
\hline Days between initial outpatient visit and hospitalization & 79 & $2(0-10)$ & 26 & $3(0-8)$ \\
\hline Jaundice & 81 & $75(93)$ & 29 & $22(76)^{*}$ \\
\hline Oliguria $†$ & 81 & $27(33)$ & 27 & $10(37)$ \\
\hline Pulmonary hemorrhage & 81 & $21(26)$ & 28 & $5(18)$ \\
\hline Respiratory distress $\ddagger$ & 81 & $20(25)$ & 29 & $11(38)$ \\
\hline Direct bilirubin & 45 & $15.8(0.8-34)$ & 18 & $7.5(0.5-32)$ \\
\hline Blood urea nitrogen $(\mathrm{mg} / \mathrm{dl})$ & 73 & $111(18-307)$ & 28 & $101(17-472)$ \\
\hline Serum creatinine $(\mathrm{mg} / \mathrm{dl})$ & 68 & $3.5(0.9-12.8)$ & 26 & $3.25(0.2-14)$ \\
\hline \multicolumn{5}{|l|}{ Hospital outcome } \\
\hline Intraperitoneal dialysis & 81 & $13(16)$ & 29 & $6(21)$ \\
\hline Intensive care unit admission & 81 & $10(12)$ & 29 & $6(21)$ \\
\hline Case fatality & 81 & $1(1)$ & 29 & $6(21)^{*}$ \\
\hline Days of hospitalization for survivors & 80 & $8(2-27)$ & 23 & $8(3-41)$ \\
\hline Serologic confirmation & 81 & & 29 & \\
\hline Paired serum samples & & $65(80)$ & & $6(21)^{*}$ \\
\hline Single serum sample & & $16(20)$ & & $19(66)^{*}$ \\
\hline No serum sample & & 0 & & $4(14)$ \\
\hline
\end{tabular}

among unconfirmed cases was due to the inability to obtain paired serum samples for diagnostic evaluation.

Interviews with 110 patients who met the surveillance definition for leptospirosis were conducted to identify the timing and sites of patients' initial contacts with the health care system. Cases procured medical attention a median of four days (range $=0-21$ days) after the onset of symptoms. Leptospirosis cases sought medical attention at 37 ambulatory services from throughout the metropolitan area, including 25 outpatient clinics and 12 hospital emergency rooms. Emergency rooms of public sector hospitals, including the infectious disease reference hospital, were reported by 58 (52\%) of 110 patients as the first medical center visited after the onset of symptoms, while public sector urgent care centers $(20 \%)$, public clinics $(14 \%)$ and private clinics $(12 \%)$ were less frequently listed. The emergency room of the infectious disease reference hospital was the site of first contact with a health service for 20 (18\%) of the leptospirosis cases (Figure 1B). The location of the next three most frequently visited medical centers and their specific catchment areas are shown in Figure 1C-E. Patients who reported seeking treatment initially at the infectious disease reference hospital or state general hospital resided throughout the city (Figure 1B and 1C), while patients who reported seeking medical attention at other hospital emergency rooms or urgent care centers resided in the respective catchment areas for these services (Figure 1D and 1E).

Cases who sought medical attention early in the course of their illness frequently presented with non-specific symptoms and received diagnoses of dengue. During the cases' first visits to a health care facility, fever (106 [99\%] of 107), generalized muscle pain (99 [93\%]), and headache (95 [89\%]) were the most commonly reported symptoms. Among the 110 interviewed cases of leptospirosis, 39 (35\%) received a diagnosis of dengue during their first visit to a medical service (Table 2). Patients who sought medical attention early in the course of illness were more likely to have received dengue diagnoses: $28(61 \%)$ of 46 patients whose initial visits occurred during the first three days of their illness received dengue diagnoses versus nine $(15 \%)$ of 59 whose visits occurred four or more days after the onset of symptoms $(\mathrm{OR}=8.6,95 \% \mathrm{CI}=3.1-24.4)$. In addition, 23 patients thought that they had dengue at the onset of their illness; therefore, 56\% (62 of 110) of all cases either received a dengue diagnosis or believed they had dengue during the early phase of their illness.

The presumptive diagnosis of dengue in the outpatient setting was based solely on symptoms and findings on physical examination since laboratory testing for dengue was not available for outpatient or emergency services in the city of 




FIGURE 1. A, map of the city of Salvador, Brazil showing the geographic distribution according to place of residence of 110 leptospirosis cases. Each dot represents a single case who met the surveillance definition for leptospirosis: shaded dots $(\bullet)$ represent laboratory-confirmed or probable cases and open circles $(\odot)$ represent unconfirmed cases who met the surveillance definition. In maps B-E, the distribution of residences $(\bullet)$ is shown for cases who procured their initial medical evaluation at four of 37 outpatient services: B, the infectious disease reference hospital (-); C, the state general hospital (+); D, a public hospital (+); E, a public urgent care center (+). Separate scale bars are shown for maps $\mathbf{A}$ and $\mathbf{B}-\mathbf{E}$.

Salvador. Cases of leptospirosis who received dengue diagnoses at their first medical evaluation were discharged and subsequently sought medical attention when their symptoms worsened. According to the triage routine in Salvador, patients with suspicion of leptospirosis or hepatitis are sent to the reference hospital, whereupon these two diseases are differentiated during clinical and laboratory evaluation. The median delay between first health service visit and arrival at the reference hospital was five days for cases initially suspected of having dengue versus 0 days for those initially suspected of having leptospirosis or hepatitis $(P<0.001)$ (Table 2). The delay in triage associated with an initial diagnosis of dengue versus leptospirosis or hepatitis was significantly longer independent of whether patients were evaluated in the first three days (median $=5$ versus 0 days; $P$ $<0.001$ ) or four or more days after the onset of symptoms (median $=3$ versus 0 days; $P<0.001$ ). Among the 39 leptospirosis cases initially suspected of having dengue, 12 (31\%) had to procure three or four health-care facilities prior to referral.

We observed a trend between delay in triage to the reference hospital and adverse outcomes among hospitalized leptospirosis cases. The delay in triage was five days (range $=0-8$ days) among deaths versus two days (range $=0-10$ ) among survivors $(P=0.08)$. Fatal cases were more likely than survivors to have been diagnosed with dengue at their first outpatient visit $(\mathrm{OR}=5.1,95 \% \mathrm{CI}=0.8-55.0)$. In addition, leptospirosis cases who required admission to the intensive care unit (ICU) were more likely to have been initially diagnosed with dengue than cases not admitted to the ICU $(\mathrm{OR}=2.7,95 \% \mathrm{CI}=0.8-9.5)$. The power of these analyses was limited because of the small sample size and difficulty in obtaining information from patients who died during hospitalization.

Cases who sought medical attention later in the course of their illness reported a higher prevalence of signs consistent with jaundice and often received diagnoses of leptospirosis or hepatitis. However, among 67 patients who reported having yellow eyes at the time of initial health service visits, $25(37 \%)$ were not triaged to the infectious disease reference hospital. Of these, 14 (56\% of 25$)$ were told that they had dengue, indicating that the dengue epidemic created a high index of suspicion even in cases presenting with an acute icteric syndrome.

\section{DISCUSSION}

Heavy rainfall over a period of several months in 1996 contributed to concurrent outbreaks of dengue and leptospirosis in the city of Salvador, Brazil. The dengue epidemic affected all parts of the city and was widely publicized through print and broadcast media. However, dengue was not responsible for deaths since cases did not develop severe forms such as dengue hemorrhagic fever. In contrast, the leptospirosis epidemic was concentrated in low-income, underserved neighborhoods. Although fewer cases were notified, leptospirosis was a significant cause of mortality: case fatality was $15 \%$ and more than 50 deaths were identified during the epidemic period. ${ }^{12}$

The present investigation was conducted in response to the failure of intravenous antibiotics and aggressive supportive therapy to prevent mortality from leptospirosis: $50 \%$ 
TABLE 2

Information obtained from interviews with 110 hospitalized leptospirosis cases, stratified according to diagnosis received at first outpatient visit

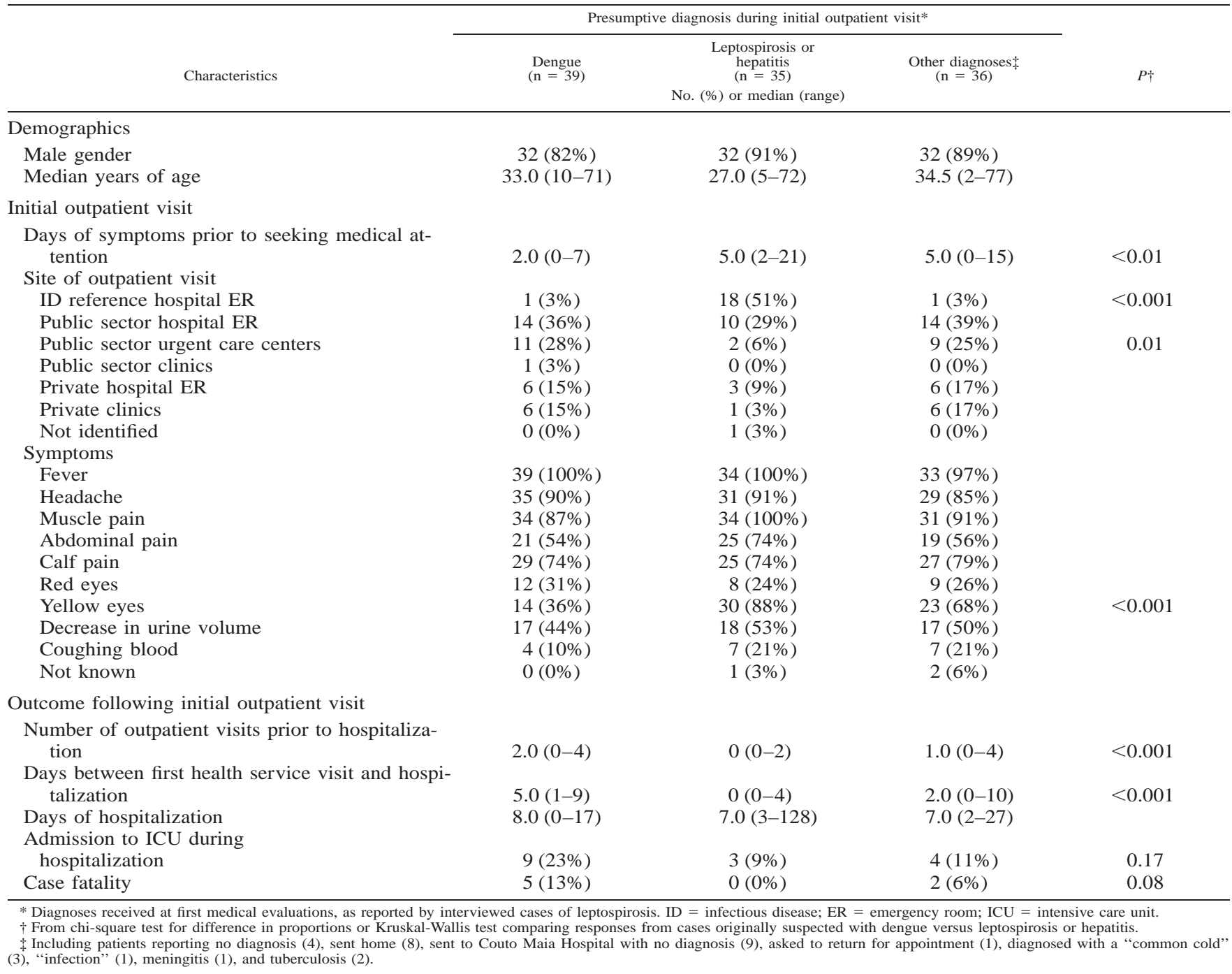

of the deaths occurred within $48 \mathrm{hr}$ of hospital admission during the 1996 epidemic. ${ }^{12}$ Leptospirosis outbreaks in dengue endemic regions of the Caribbean and Central Ameri$\mathrm{ca}^{2,19,20}$ have raised awareness concerning diagnostic confusion between leptospirosis and dengue and its potential impact on outcome for leptospirosis. During dengue surveillance in Puerto Rico in 1996, leptospirosis was established as the diagnosis in 21 laboratory-unconfirmed cases of dengue and among these, 29\% had severe clinical presentations and one patient died. ${ }^{2}$ Furthermore, leptospirosis was found retrospectively to be the cause in $31 \%$ of cases who died of suspected dengue. ${ }^{2}$ In Barbados, Levett and others ${ }^{21}$ demonstrated significant misdiagnosis during surveillance for both leptospirosis and dengue: more than $30 \%$ of all cases identified as suspected leptospirosis had dengue IgM antibodies and $7 \%$ of laboratory-unconfirmed cases of suspected dengue had leptospiral IgM antibodies. During the same period, an increase in mortality from leptospirosis was observed and attributed to a large concurrent outbreak of dengue. $^{21}$
The present study is the first to prospectively investigate the effect of a dengue epidemic on the referral of leptospirosis cases and on subsequent outcomes. In Salvador, a high index of suspicion for dengue in outpatient facilities contributed to a significant delay in the triage of leptospirosis cases to the infectious disease reference hospital. This delay was longest for patients who presented in the early phase of their illness and received the diagnosis of dengue. Patients who sought medical attention later in the course of illness were more likely to receive diagnoses of leptospirosis or hepatitis and to be promptly triaged to the reference hospital. In addition, a trend was observed between receiving a dengue diagnosis in outpatient facilities and adverse outcomes during hospitalization, including higher case fatality and admission to the intensive care unit. These findings strongly suggest that diagnostic confusion with dengue had an impact on outcome for leptospirosis.

In this investigation, information was analyzed from patients who met the surveillance case definition of leptospirosis. These included unconfirmed cases (26\% of 110) for 
the majority of which complete diagnostic testing could not be performed due to the lack of paired serum samples. However, in patients from whom paired samples were obtained, the surveillance case definition had a positive predictive value of $92 \%$. Cases with or without laboratory evidence showed no differences in the site of outpatient visits, the timing of first contact with the health-care system, or geographic distribution. Therefore, the inclusion of unconfirmed cases in the analysis does not appear to be a likely source of bias. A limitation of the present study was the use of patient recall to obtain information on presumptive diagnoses and symptoms at the time of initial outpatient evaluation. We observed that $94 \%$ of the patients (104 of 110) recalled their presumptive diagnosis and that re-constructed timelines of onset of specific symptoms correlated with classic "earlyphase" and "late-phase" manifestations of leptospirosis.

We identified a "window of opportunity" during which a significant proportion of leptospirosis cases procured medical attention in the initial phase of their illness (42\% in the first three days). It is within this period that oral doxycyline therapy has been shown in a randomized clinical trial to provide benefit in reducing morbidity from leptospirosis. ${ }^{13}$ Observational studies have suggested that the increased use of empiric antibiotic treatment for febrile patients during epidemic periods contributed to the gradual decrease in the case fatality rate for leptospirosis observed in New Caledonia $^{22}$ and in Barbados. ${ }^{21,23}$ However, in the present study, early-phase leptospirosis was confused with dengue during this critical period. In Salvador, antibiotic treatment was not administered until patients were admitted to the reference hospital, and only $5 \%$ of the interviewed patients were hospitalized within the first three days of symptoms.

As observed in studies from other settings,,$^{2,19-21}$ the lack of specific clinical markers for early-phase leptospirosis hinders its differentiation from dengue. This is especially important in developing country settings, such as in the present study, in which diagnosis at ambulatory health services are often based solely on clinical findings. Therefore, an inexpensive and rapid laboratory test is needed for ambulatorybased diagnosis. Screening for leptospirosis among febrile outpatients requires a highly sensitive and specific test, since the overall prevalence of this disease among ambulatory patients with acute febrile illnesses is expected to be low. Rapid serologic tests such as the Lepto-dipstick ${ }^{24-26}$ and latex agglutination assay, ${ }^{27}$ have been developed, but the reported sensitivities of these tests have been approximately $50 \%$ for identifying cases in the first 10 days of symptoms. Recently, the Lepto-dipstick assay was re-evaluated and found to have a sensitivity of $85 \%$ for early-phase samples, ${ }^{26}$ but the observed specificity (88\%) would result in a high proportion of false-positive results in an ambulatory setting.

Misidentification of leptospirosis during the outbreak was not limited to diagnosis during the early phase of illness. We observed that $37 \%$ of leptospirosis cases with the late-stage sign of jaundice at the time of first contact with the healthcare system were not triaged promptly to the reference hospital. Of these, $56 \%$ were misdiagnosed as dengue, despite jaundice being an uncommon finding in dengue patients. ${ }^{28}$ Paradoxically, $18 \%$ of the interviewed cases procured initial medical assistance at the infectious disease hospital. These cases resided in regions throughout the city, and not pref- erentially in proximity to the hospital (Figure 1B) and had a high proportion of severe complications such as jaundice during emergency room evaluation. These findings indicate that a part of the population of Salvador is familiar with the late-phase manifestations of leptospirosis and the need for self-referral to the reference hospital. Therefore, dissemination of information concerning the signs and symptoms of leptospirosis to health care professionals and the public may be an effective intervention to aid case identification during annual urban epidemics.

The dengue epidemic had an impact not only on leptospirosis case identification but also on the operation of public sector emergency services. More than 20,000 outpatients with dengue during the epidemic season sought acute medical care at these facilities, impairing the ability of health care staff to effectively evaluate patients with leptospirosis as well as other diseases. As dengue continues to spread to urban centers throughout the developing world, ${ }^{16}$ diagnostic confusion between dengue and leptospirosis will increasingly affect the early case identification and initiation of antibiotic therapy needed to reduce mortality and morbidity from leptospirosis.

Acknowledgments: We thank the team of clinicians and staff at Couto Maia Hospital, especially Ana Maia and Adair de Paiva Barreto, and Suzana Ramos Ferrer at CPqGM for the laboratory confirmation of leptospirosis cases; and the medical residents of Weill Medical College of Cornell University (Donald Curran, LaMar Hasbrouck, and Michelle Floris) for database management.

Financial support: This study was supported by Fundação Oswaldo Cruz/Brazilian Ministry of Health (0250.250.415), the Brazilian National Research Council (52.1229/98-7, 300.861/96-6, and 350.052/ 95-6, and FINEP 4196086200), and the National Institutes of Health (TW00905, D43 TW00919, AI-30639, and KO8 AI-01605).

Authors'addresses: Brendan Flannery and Lee W. Riley, School of Public Health, University of California, 140 Warren Hall, Berkeley, CA 94720-7360. Mônica M. Pereira and Cibele M. Ribeiro Dourado, Hospital Couto Maia, Secretary of Health for the State of Bahia, Salvador, Bahia, Brazil. Luciana de Freitas Velloso, Clarissa de Castro Carvalho, Lina Góes de Codes, Giovanna de Saboia Orrico, and Mitermayer G. Reis. Centro de Pesquisas Gonçalo Moniz, FIOCRUZ, R. Waldemar Falcão, 121 Brotas, 40295-001 Salvador, Bahia, Brazil. Albert I. Ko, Weill Medical College of Cornell University, 1300 York Ave A-431, New York, NY 10021

Reprint requests: Albert I. Ko, Centro de Pesquisas Gonçalo Moniz, FIOCRUZ, R. Waldemar Falcão, 121 Brotas, 40295-001 Salvador, Bahia, Brazil.

\section{REFERENCES}

1. Faine S, Adler B, Bolin C, Perolat P, 1999. Leptospira and Leptospirosis. Second edition. Melbourne, Australia: MediSci.

2. Sanders EJ, Rigau-Pérez JG, Smits HL, Deseda CC, Vorndam VA, Aye T, Spiegel RA, Weyant RS, Bragg SL, 1999. Increase of leptospirosis in dengue-negative patients after a hurricane in Puerto Rico in 1996. Am J Trop Med Hyg 61: 399404.

3. Bounlu K, Insisiengmay S, Vanthanouvong K, Saykham, Widjaja S, Iinuma K, Matsubayashi K, Laras K, Putri MP, Endy TP, Vaughn DW, Raengsakulrach B, Hyams KC, Hayden M, Scheffel C, Corwin AL, 1998. Acute jaundice in Vientiane, Lao People's Democratic Republic. Clin Infect Dis 27: 717721.

4. Farr WR, 1995. Leptospirosis. Clin Infect Dis 21: 1-8.

5. Yersin C, Bovet P, Mérien F, Clément J, Laille M, Van Ranst M, Perolat P, 2000. Pulmonary haemorrhage as a predominant 
cause of death in leptospirosis in Seychelles. Trans $R$ Soc Trop Med Hyg 94: 71-76.

6. Marotto PCF, Nascimento CMR, Eluf-Neto J, Marotto MS, Andrade L, Sztajnbok J, Seguro AC, 1999. Acute lung injury in leptospirosis: clinical and laboratory features, outcome and factors associated with mortality. Clin Infect Dis 29: 15611563.

7. Lomar AV, Diament D, Torres JR, 2000. Leptospirosis in Latin America. Infect Dis Clin North Am 14: 23-39.

8. Corrêa MOA, 1975. Human leptospirosis in Brazil. Int J Zoonoses 2: 1-9.

9. Rios-Gonçalves AJ, Capone D, Paz NA, Paulo RVV, Dias TBCM, Lago VCC, Carvalho LMA, 1990. Leptospirose: observações sobre as mudanças dos padrões clínicos no Rio de Janeiro após a grande epidemia de 1988. Arq Bras Med 64. 389-397.

10. Azevedo R, Corrêa MOA, 1968. Considerações em tôrno da epidemia de leptospirose na cidade de Recife em 1966. Aspectos epidemiológicos, laboratoriais e clinicos. Rev Inst Adolfo Lutz 28: 85-111.

11. Caldas EM, Sampaio MB, Costa E, Miranda G, 1979. Estudo epidemiológico de surto de leptospirose ocorrido na cidade do Salvador, Bahia, em maio e junho de 1978. Rev Inst Adolfo Lutz 39: 85-94.

12. Ko AI, Reis MG, Dourado CMR, Johnson WD, Riley LW, and the Salvador Leptospirosis Study Group, 1999. Urban epidemic of severe leptospirosis in Brazil. Lancet 354: 820-825.

13. McClain BL, Ballou WR, Harrison SM, Steinweg DL, 1984 Doxycycline therapy for leptospirosis. Ann Intern Med 100: 696-698.

14. Edwards CN, Nicholson GD, Hassell TA, Everard COR, Callender J, 1988. Penicillin therapy in icteric leptospirosis. Am J Trop Med Hyg 39: 388-390.

15. Watt G, Tuazon ML, Santiago E, Padre LP, Calubaquib C, Ranoa CP, Laughlin LW, 1988. Placebo-controlled trial of intravenous penicillin for severe and late leptospirosis. Lancet 1: 433-435.

16. World Health Organization, 2000. Dengue/dengue haemorrhagic fever: situation in 2000. Wkly Epidemiol Rec 75: 193-200.

17. Castro JSM, 1997. Dengue. Bol Epidemiol SESAB 5: 2.

18. World Health Organization, 1982. Faine S, ed. Guidelines for the Control of Leptospirosis. Geneva: World Health Organization.
19. Zaki SR, Shieh WJ, 1996. Leptospirosis associated with outbreak of acute febrile illness and pulmonary hemorrhage, Nicaragua, 1995. Lancet 347: 535-536.

20. Trevejo RT, Rigau-Pérez JG, Ashford DA, McClure EM, Jarquín-González C, Amador JJ, Reyes JO, Gonzalez Alcides, Zaki SR, Shieh WJ, McLean RG, Nasci RS, Weyant RS, Bolin CA, Bragg SL, Perkins BA, Spiegel RA, 1998. Epidemic leptospirosis associated with pulmonary hemorrhage-Nicaragua, 1995. J Infect Dis 178: 1457-1463

21. Levett PN, Branch SL, Edwards CN, 2000. Detection of dengue infection in patients investigated for leptospirosis in Barbados. Am J Trop Med Hyg 62: 112-114

22. Merien F, Perolat P, 1996. Public health importance of human leptospirosis in the South Pacific: a five-year study in New Caledonia. Am J Trop Med Hyg 55: 174-178.

23. Everard COR, Edwards CN, Everard JD, Carrington DG, 1995. A twelve-year study of leptospirosis on Barbados. Eur J Epidemiol 11: 311-320.

24. Gussenhoven GC, van der Hoorn MAWG, Goris MGA, Terpatra WJ, Hartskeerl RA, Mol BW, van Ingen CW, Smits HL, 1997. LEPTO dipstick, a dipstick assay for detection of Leptospiraspecific immunoglobulin $\mathrm{M}$ antibodies in human sera. J Clin Microbiol 35: 92-97.

25. Smits HL, Ananyina YV, Chereshsky A, Dancel L, Lai-A-Fa RFM, Chee HD, Levett PN, Masuzawa T, Yanagihara Y, Muthusethupathi MA, Sanders EJ, Sasaki DM, Domen H, Yersin C, Aye T, Bragg SL, Gussenhoven GC, Goris MGA, Terpstra WJ, Hartskeerl RA, 1999. International multicenter evaluation of the clinical utility of a dipstick assay for detection of leptospira-specific immunoglobulin $\mathrm{M}$ antibodies in human serum specimens. J Clin Microbiol 37: 2904-2909.

26. Smits HL, Hartskeerl RA, Terpstra WJ, 2000. International multi-centre evaluation of a dipstick assay for human leptospirosis. Trop Med Int Health 5: 124-128.

27. Smits HL, van der Hoorn MAWG, Goris MGA, Gussenhoven GC, Yersin C, Sasaki DM, Terpstra WJ, Hartskeerl RA, 2000. Simple latex agglutination assay for rapid serodiagnosis of human leptospirosis. J Clin Microbiol 38: 1272-1275.

28. Kalayanarooj DW, Vaughn DW, Nimmannitya S, Green S, Suntayakorn S, Kunentrasai N, Viramitrachai W, Ratanachu-eke S, Kiatpolpoj S, Innis BL, Rothman AL, Nisalak A, Ennis FA, 1997. Early clinical and laboratory indicators of acute dengue illness. J Infect Dis 176: 313-321. 